PROCEEDINGS OF THE

AMERICAN MATHEMATICAL SOCIETY

Volume 134, Number 8, Pages 2359-2362

S 0002-9939(06)08366-3

Article electronically published on February 6, 2006

\title{
ON THE UNIFORM CONVEXITY OF $L^{p}$
}

\author{
HARALD HANCHE-OLSEN
}

(Communicated by Jonathan M. Borwein)

\begin{abstract}
We present a short, direct proof of the uniform convexity of $L^{p}$ spaces for $1<p<\infty$.
\end{abstract}

The standard proof of the uniform convexity of $L^{p}$ using Clarkson's [1] or Hanner's [2] inequalities (see also [4]) is rarely taught in functional analysis classes, in part (the author imagines) because the proofs of those inequalities are quite nonintuitive and unwieldy. Here we present a direct proof, cheerfully sacrificing the optimal bounds - for which, see [2, 4].

In order to motivate our proof, and to explain how it might fit into a standard functional analysis course, we remind the reader of Young's inequality that

$$
\operatorname{Re} u v \leq \frac{|u|^{p}}{p}+\frac{|v|^{q}}{q}, \quad 1<p<\infty, \frac{1}{p}+\frac{1}{q}=1 .
$$

This has many proofs. For example, if $u, v>0$ we write $u=e^{s / p}$ and $v=e^{t / q}$, and use the convexity of the exponential function to arrive at $e^{s / p+t / q} \leq e^{s} / p+e^{t} / q$, which is (11). The general case follows immediately. Moreover, this proof shows that the inequality in (1) is strict unless $u v \geq 0$ and $|u|^{p}=|v|^{q}$, in which case $u v=|u|^{p}=|v|^{q}$.

From this one can proceed to prove the Hölder inequality in the normalized case:

$$
\operatorname{Re} \int_{\Omega} u v d \mu \leq 1, \quad\|u\|_{p}=\|v\|_{q}=1
$$

with equality if and only if $u v=|u|^{p}=|v|^{q}$ a.e.

Recall that a normed space $X$ is called uniformly convex [1] if for each $\varepsilon>0$ there is some $\delta>0$ so that $x, y \in X,\|x\| \leq 1,\|y\| \leq 1$ and $\|x+y\|>2-\delta$ imply $\|x-y\|<\varepsilon$.

Perhaps a bit more intuitive is the following equivalent condition, which we might call thin slices of the unit ball are small: Given $\varphi \in X^{*}$ with $\|\varphi\|=1$, define the $\delta$-slice $S_{\varphi, \delta}=\{x \in X:\|x\| \leq 1$ and $\operatorname{Re} \varphi(x)>1-\delta\}$. The "thin slices" condition states that for each $\varepsilon>0$ there is some $\delta>0$ so that, if $\varphi \in X^{*}$ with $\|\varphi\|=1$, then $\|x-y\|<\varepsilon$ for all $x, y \in S_{\varphi, \delta}$.

This condition follows trivially from uniform convexity. The proof of the converse requires a minor trick: Given $x, y \in X$ with $\|x\| \leq 1,\|y\| \leq 1$ and $\|x+y\|>2-\delta$, pick $\varphi \in X^{*}$ with $\|\varphi\|=1$ and $\operatorname{Re} \varphi(x+y)>2-\delta$. Then $\operatorname{Re} \varphi(x)=\operatorname{Re} \varphi(x+y)-$

Received by the editors March 9, 2005

2000 Mathematics Subject Classification. Primary 46E30.

(c)2006 Harald Hanche-Olsen 
$\operatorname{Re} \varphi(y)>2-\delta-1=1-\delta$, and similarly for $y$. If $\delta$ was chosen according to the "thin slices" condition, $\|x-y\|<\varepsilon$ follows.

It should be noted that for the above proof to work, it may be sufficient to prove the "thin slices" condition for some functionals. In fact, we shall only use functionals on $L^{p}$ arising from functions in $L^{q}$, without using the knowledge that all functionals on $L^{p}$ are of this form.

If we let $\delta \rightarrow 0$, then $S_{\varphi, \delta}$ shrinks to the set of unit vectors satisfying $\operatorname{Re} \varphi(x)=1$, and the "thin slices" condition guarantees that there can be only one such $x$. In the $L^{p}$ case, this corresponds to the uniqueness, given $v \in L^{q}$, of a unit vector $u \in L^{p}$ yielding equality in Hölder's inequality.

Since this uniqueness came about due to the sharp inequality in (11), it is reasonable to expect that an improved lower estimate on the difference between the two sides of (11) might yield a version of the "thin slices" condition.

This is precisely what the present proof does. In fact, we shall show that given $\varepsilon>0$, a single $\delta$ will suffice for all $L^{p}$ spaces (for a fixed $p$ ).

The requisite inequality turns out to be, in general, quite difficult to find, so we concentrate on the case $v=1$ in (1) and use the improved inequality to show the "thin slices" condition for the functional arising from the constant function 1 on $L^{p}$ over a probability space. Afterwards, in Lemma 2, we show that this is sufficient to cover the general case.

Lemma 1. Given $1<p<\infty$ and $\varepsilon>0$, there exists $\delta>0$ so that, for every probability space $(\Omega, \nu)$ and every measurable function $z$ on $\Omega,\|z\|_{p} \leq 1$ and $\operatorname{Re} \int_{\Omega} z d \nu>1-\delta$ imply $\|z-1\|_{p}<\varepsilon$.

Proof. Consider the function

$$
f(u)=|u|^{p}-1+p(1-\operatorname{Re} u)
$$

and note that $f(u)>0$ everywhere except for the value $f(1)=0$. (This is the case $v=1$ in (11).) Further, note that $f(u)$ and $|u-1|^{p}$ are asymptotically equal as $|u| \rightarrow \infty$. Thus, given $\varepsilon>0$, we can find some $\alpha>1$ so that

$$
|u-1|^{p} \leq \alpha f(u) \text { whenever }|u-1| \geq \varepsilon .
$$

Assume that $z$ satisfies the stated conditions, and let $E=\{\omega \in \Omega:|z(\omega)-1|<\varepsilon\}$. Then

$$
\begin{aligned}
\|z-1\|_{p}^{p} & =\int_{E}|z-1|^{p} d \nu+\int_{\Omega \backslash E}|z-1|^{p} d \nu \\
& \leq \varepsilon^{p}+\alpha \int_{\Omega} f(z) d \nu \\
& \leq \varepsilon^{p}+p \alpha\left(1-\int_{\Omega} \operatorname{Re} z d \nu\right) \\
& <\varepsilon^{p}+p \alpha \delta .
\end{aligned}
$$

Thus picking $\delta=\varepsilon^{p} /(p \alpha)$ is sufficient to guarantee $\|z-1\|_{p}<2^{1 / p} \varepsilon$.

Lemma 2. Given $1<p<\infty, p^{-1}+q^{-1}=1$ and $\varepsilon>0$, there exists $\delta>0$ so that the following holds: If $u, w$ are measurable functions on a measure space $\Omega$ with $\|u\|_{p} \leq 1$ and $\|w\|_{q}=1$ and $\int_{\Omega} \operatorname{Re} u w d \mu>1-\delta$, then $\|u-v\|_{p}<\varepsilon$, where $v$ is the function satisfying $v w=|v|^{p}=|w|^{q}$ a.e. 
Proof. Let $p$ and $\varepsilon$ be given, and choose $\delta$ as in Lemma 1 .

Let $u, v$ and $w$ be as stated above. Since nothing is changed by multiplying $u$, $v$ by a complex function of absolute value 1 , and dividing $w$ by the same function, we may assume without loss of generality that $v \geq 0$ and $w \geq 0$.

Let $z=u / v$ where $v \neq 0$ and let $z=0$ where $v=0$. Thus $z v=u$ where $v \neq 0$ and $z v=0$ where $v=0$. Since $(u-z v) z v=0$ we find $\|u\|_{p}^{p}=\|u-z v\|_{p}^{p}+\|z v\|_{p}^{p}$. Also $\operatorname{Re} \int_{\Omega} z v w d \mu=\operatorname{Re} \int_{\Omega} u w d \mu>1-\delta$, so $\|z v\|_{p}>1-\delta$ and $\|u-z v\|_{p}^{p}<1-(1-\delta)^{p}$.

Let $\nu$ be the probability measure

$$
d \nu=v w d \mu=v^{p} d \mu=w^{q} d \mu .
$$

We find

$$
\int_{\Omega}|z|^{p} d \nu=\int_{v \neq 0}|u|^{p} d \mu \leq 1, \quad \operatorname{Re} \int_{\Omega} z d \nu=\operatorname{Re} \int_{\Omega} u w d \mu>1-\delta .
$$

By Lemma 1, we now get

$$
\varepsilon^{p}>\int_{\Omega}|z-1|^{p} d \nu=\int_{\Omega}|z-1|^{p} v^{p} d \mu=\int_{v \neq 0}|u-v|^{p} d \mu .
$$

On the other hand,

$$
\int_{v=0}|u-v|^{p} d \mu=\int_{\Omega} \mid\left(u-\left.z v\right|^{p} d \mu<1-(1-\delta)^{p} .\right.
$$

We therefore get $\|u-v\|_{p}^{p}<\varepsilon+1-(1-\delta)^{p}$, and the proof is complete.

Theorem 3 (Clarkson). $L^{p}$ is uniformly convex when $1<p<\infty$.

Proof. Consider $x, y \in L^{p}$ with $\|x\|_{p}=\|y\|_{p}=1$ and $\|x+y\|_{p}>2-\delta$. Let $v=(x+y) /\|x+y\|_{p}$, and choose $w \in L^{q}$ with $v w=|v|^{p}=|w|^{q}$. In particular $\|v\|_{p}=\|w\|_{q}=1$. Then

$$
\int_{\Omega}(x+y) w d \mu=\|x+y\|_{p} \int_{\Omega} v w d \mu=\|x+y\|_{p}>2-\delta .
$$

Also, since $\operatorname{Re} \int_{\Omega} y w d \mu \leq 1$, this implies $\operatorname{Re} \int_{\Omega} x w d \mu>1-\delta$. If $\delta$ is chosen according to Lemma 2 , we get $\|x-v\|_{p}<\varepsilon$. Similarly $\|y-v\|_{p}<\varepsilon$, and so $\|x-y\|_{p}<2 \varepsilon$.

One reason for our interest in including the uniform convexity in a standard functional analysis class is that this implies the reflexivity of these spaces, by the Milman-Pettis theorem. (For a remarkably brief proof, see 3.) This, in turn, can be used to prove the standard duality theorem for $L^{p}$ and $L^{q}$. Of course, this requires some comparatively heavy machinery, but it is machinery that is usually included in such classes anyway.

We finish by outlining the proof. If $v \in L^{p}$, then $\|v\|_{p}=\max _{\|w\|_{q}=1} \operatorname{Re} \int_{\Omega} v w d \mu$. This is part Hölder's inequality, and part - assuming we normalize $v$ - the choice of $w$ satisfying $v w=|v|^{p}=|w|^{q}$. Thus, with the standard duality, $L^{q}$ is isometrically embedded in $\left(L^{p}\right)^{*}$. Now assuming that $L^{q} \neq\left(L^{p}\right)^{*}$, an appeal to the Hahn-Banach theorem produces a nonzero bounded linear functional $f$ on $\left(L^{p}\right)^{*}$ which vanishes on $L^{q}$. Since $L^{p}$ is reflexive, $\varphi$ is of the form $\varphi(y)=y(u)$ for some $u \in L^{p}$. In particular, for each $w \in L^{q}$ we get $0=\varphi(w)=\int_{\Omega} u w d \mu$. But then $u=0$, which is a contradiction. 


\section{REFERENCES}

[1] J. A. Clarkson, Uniformly convex spaces, Trans. Amer. Math. Soc. 40 (1936), no. 3, 396-414. MR 1501880

[2] O. Hanner, On the uniform convexity of $L^{p}$ and $l^{p}$, Ark. Mat. 3 (1956), 239-244. MR0077087 $(17: 987 \mathrm{~d})$

[3] J. R. Ringrose, A note on uniformly convex spaces, J. London Math. Soc. 34 (1959), 92. MR0100215 (20:6648)

[4] K. Ball, E. A. Carlen and E. H. Lieb, Sharp uniform convexity and smoothness inequalities for trace norms, Invent. Math. 115 (1994), no. 3, 463-482. MR1262940 (95e:47027)

Department of Mathematical Sciences, Norwegian University of Science and TechNOLOGY, NO-7491 TRONDHEIM, NORWAY

E-mail address: hanche@math.ntnu.no

URL: http://www. math.ntnu.no/ $\sim_{\text {hanche/ }}$ 